\title{
Silent brain infarct is independently associated with arterial stiffness indicated by cardio-ankle vascular index (CAVI)
}

\begin{abstract}
Naoki Saji ${ }^{1,2}$, Kazumi Kimura ${ }^{1}$, Hirotaka Shimizu ${ }^{2}$ and Yasushi Kita ${ }^{2}$
It is still unclear whether silent brain infarct (SBI) and white-matter hyperintensities (WMHs) on magnetic resonance imaging (MRI) scans are associated with cardio-ankle vascular index (CAVI), a novel parameter of arterial stiffness. We studied 220 consecutive patients (mean age, 69 years) without a history of stroke or transient ischemic attack. Patients were assessed for the presence of SBI, WMHs and risk factors. Arterial stiffness was evaluated using CAVI. Patients were categorized into one of two groups according to the presence or absence of SBI and WMHs, and clinical characteristics were compared between the two groups. CAVI was significantly higher in patients with SBI or in patients with WMHs than in those without those respective findings. The CAVI cutoff values for detection of SBI and WMHs were 9.2 and 8.9 , respectively. On multivariable analyses, CAVI, a one point increase in CAVI: odds ratio (OR), 1.25; 95\% confidence interval (Cl), 1.01-1.56; CAVI $\geqslant 9.2$ : OR, 2.34; 95\% Cl, 1.165.02, was independently associated with SBI, however, CAVI was not independently associated with WMHs. Patients with CAVI $\geqslant 9.2$ had higher OR for the presence of both $\mathrm{SBI}$ and WMHs (OR, 2.57; 95\% Cl, 1.15-5.98) when compared with patients with CAVI $<9.2$ after adjustment for age and sex. SBI is independently associated with arterial stiffness indicated by CAVI. Hypertension Research (2012) 35, 756-760; doi:10.1038/hr.2012.20; published online 1 March 2012
\end{abstract}

Keywords: arterial stiffness; cardio-ankle vascular index; cerebral small vessel disease; silent brain infarct; white-matter hyperintensities

\section{INTRODUCTION}

Silent brain infarct (SBI) may represent a risk factor for stroke and cerebral small vessel diseases (SVDs) and is associated with cognitive impairment and poor functional outcome after stroke. ${ }^{1-14}$ Risk factors for SVD include age, hypertension, coronary artery calcification and chronic kidney disease. ${ }^{7-16}$ Likewise, white-matter hyperintensities (WMHs) are associated with cognitive impairment, executive function deficits and gait disturbance. ${ }^{7-11,16-18}$ The etiology of SBI, a cerebral arterial occlusive lesion, is related to mainly arteriolosclerosis such as lipohyalinosis, microatheroma and fibrinoid necrosis, while the etiology of WMHs is related to cerebral disruption of the blood-brain barrier or leakage of plasma due to SVD or cerebral microvascular arteriosclerosis. ${ }^{8-13}$ Therefore, both SBI and WMHs may be associated with arterial stiffness.

Cardio-ankle vascular index (CAVI) is a relatively novel parameter that can be used to assess arterial stiffness independent of blood pressure. ${ }^{8,19-24}$ Arterial stiffness, as measured by conventional carotidfemoral pulse wave velocity (PWV) measurement, aortic PWV measurement or non-invasive brachial-ankle PWV (baPWV) measurement, is associated with microvascular early atherosclerotic changes and is an independent predictor for cardiovascular events and deaths. ${ }^{1-3,7,13,14,25-29}$ Furthermore, baPWV can be used to assess for the presence of SBI and WMHs. ${ }^{7,13,14,21,30}$ However, the relationship between SVD and CAVI is not yet clear.

The aim of this study was to investigate whether SBI and WMHs in elderly patients are associated with arterial stiffness, as measured by CAVI.

\section{METHODS}

Subjects

This was a single-center observational study approved by the Institutional Review Board at the Hyogo Brain and Heart Center at Himeji. Between April 2010 and September 2011, we consecutively enrolled patients who visited the clinic in the Department of Neurology at the Hyogo Brain and Heart Center at Himeji to request medical evaluation for a routine checkup or for assessment of possible cerebrovascular disease. Patients were enrolled in this study provided that none of the following were present: history of stroke, transient ischemic attack or dementia; abnormality on neurological examination; or evidence of atrial fibrillation, atrial flutter, symptomatic and/or treated peripheral arterial disease. Informed consent was obtained from all patients. All magnetic resonance imaging (MRI) scans and other measurements were assessed by clinicians who were blind to any clinical information. 


\section{MRI assessment}

All patients underwent assessment by $1.5 \mathrm{~T}$ brain MRI (Intera, Philips Medical Systems, Best, The Netherlands), including diffusion-weighted imaging, fluid-attenuated inversion recovery (FLAIR) imaging and T2-weighted imaging (T2WI). Image acquisition parameters for MRI were as described previously. ${ }^{4,7,14}$ SBI was defined as a focal lesion of at least $3 \mathrm{~mm}$ in diameter, with hyperintensity on T2WI and hypointensity on FLAIR. ${ }^{5-7,14}$ WMHs were defined as an irregular periventricular hyperintensity (Fazekas grade $\geqslant 3$ ) and/ or early confluent or confluent separate deep white-matter hyperintense lesions (Fazekas grade $\geqslant 2$ ) on T2WI and FLAIR, based on the rating scales of ischemic tissue damage due to arteriosclerosis. ${ }^{5,7,14,31,32}$

\section{Carotid ultrasonography}

Far wall common carotid artery intima-media thickness (CCAIMT) was measured using images acquired by high-resolution B-mode ultrasonography with a 7.5-MHz linear array transducer. ${ }^{14}$ Three determinations of intima-media thickness were performed at the thickest point: maximum CCAIMT and two adjacent points $(1 \mathrm{~cm}$ upstream and $1 \mathrm{~cm}$ downstream from this site). These three determinations were averaged (mean CCAIMT). ${ }^{33}$ Mean CCAIMT was determined on both the left and right sides, and the larger value was used for analyses. $^{14}$

\section{Measurement of ankle-brachial pressure index and CAVI}

Ankle-brachial pressure index and CAVI were measured using an oscillometric device (VaSera VS-1500; Fukuda Denshi, Co Ltd., Tokyo, Japan), which has been described previously. ${ }^{8,19-23}$ After examinations had been performed on the right and left sides, the lower ankle-brachial pressure index and the higher CAVI were selected for analyses. Ankle-brachial pressure index $<0.9$ was considered as indicative of peripheral arterial disease. ${ }^{7}$

\section{Risk factors}

Hypertension was defined as systolic blood pressure $\geqslant 140 \mathrm{~mm} \mathrm{Hg}$ or diastolic blood pressure $\geqslant 90 \mathrm{~mm} \mathrm{Hg}$ and/or by the use of antihypertensive agents. Hypercholesterolemia was defined as serum total cholesterol level $\geqslant 220 \mathrm{mg} \mathrm{dl}^{-1}$ and/or by the use of statins. Diabetes mellitus was defined as hemoglobin Alc level $\geqslant 6.5 \%$ and/or by the use of oral hypoglycemic agents or insulin, and/or a serum fasting blood sugar level $\geqslant 126 \mathrm{mg} \mathrm{dl}^{-1}$. Ischemic heart disease (IHD) was defined as a history of a cardiologist-diagnosed angina pectoris, evidence of prior myocardial infarction or a prior coronary revascularization procedure (percutaneous coronary intervention or coronary artery bypass surgery). Current smoking habits were recorded.

\section{Statistical analysis}

Continuous variables are expressed as mean values and s.d. and were compared using unpaired Student's $t$-tests. Variables exhibiting skewed distributions are expressed as medians and inter-quartile range and were compared using the Wilcoxon's rank-sum test. Categorical variables are expressed as frequencies and percentages and compared using $\chi^{2}$-tests. Potential variables with $P<0.10$ on a univariate analysis were entered into a stepwise logistic regression analysis, which was performed to identify the variables independently associated with SBI and WMHs. Receiver operating characteristic curve analysis was performed to determine the cutoff values of CAVI for the presence of SBI and WMHs. A two-tailed $P<0.05$ was considered to indicate statistical significance. Odds ratios (ORs) are presented with $95 \%$ confidence intervals (95\% CIs). Data were analyzed using the JMP 8.0.2 software package (SAS Institute Inc., Cary, NC, USA).

\section{RESULTS}

\section{Patient characteristics}

A total of 220 patients were included in this study. Table 1 summarizes the characteristics of these patients. A total of 168 patients $(76 \%)$ had hypertension, 102 patients (46\%) had WMHs and 54 patients $(25 \%)$ had SBI. Among the 54 patients with SBI, 43 had both SBI and WMHs and 15 had multiple infarcts. All of 15 patients with multiple infarcts also had WMHs. Distribution of the SBI was as follows: basal ganglia
(54\%), thalamus $(14 \%)$, centrum semiovale $(10 \%)$, corona radiata $(8 \%)$, internal capsule (5\%), cerebellum hemisphere (4\%), cortical lobe $(3 \%)$ and pons $(2 \%)$.

\section{Comparison between patients with/without SBI}

Table 2 summarizes the clinical characteristics of patients with or without SBI. Patients with SBI were significantly older, had increased CAVI and CCAIMT, and were more likely to be female and have hypertension or IHD when compared with patients without SBI (Table 2). The CAVI cutoff value for detection of SBI was 9.2, which was associated with a $76 \%$ sensitivity and $63 \%$ specificity (AUC (area under the receiver operating characteristic curve $)=0.67 \pm 0.05,95 \%$ CI, 0.58-0.76; $P<0.001$ ). In multivariable analyses (Table 3), IHD (OR, 2.74; 95\% CI, 1.36-5.57), CAVI (every one point increase: OR, 1.25 ; 95\% CI, 1.01-1.56) and age (OR, 1.08; 95\% CI, 1.03-1.13) were independently associated with SBI (model 1). The possibility of the presence of SBI was significantly increased in patients with CAVI $\geqslant 9.2$ (multivariable-adjusted OR, 2.34; 95\% CI, 1.16-5.02) with the same adjustment applied (model 2).

\section{Table 1 Patient characteristics}

\begin{tabular}{lc}
\hline & All subjects ( $\mathrm{n}=220)$ \\
\hline Female sex & $89(40 \%)$ \\
Age, years & $69(10)$ \\
$\mathrm{ABI}$ & $1.09(1.04-1.14)$ \\
$\mathrm{CAVI}$ & $9.1(8.1-9.8)$ \\
CCAIMT, mm & $1.5(1.1-1.9)$ \\
WMHs & $102(46 \%)$ \\
SBI & $54(25 \%)$ \\
Hypertension & $168(76 \%)$ \\
Hypercholesterolemia & $108(49 \%)$ \\
Diabetes mellitus & $61(25 \%)$ \\
Ischemic heart disease & $63(29 \%)$ \\
Smoking & $37(17 \%)$
\end{tabular}

Abbreviations: $\mathrm{ABI}$, ankle-brachial pressure index; $\mathrm{CAVI}$, cardio-ankle vascular index; CCAIMT, common carotid artery intima-media thickness; SBI, silent brain infarct; WMHs, white-matter hyperintensities.

Data are mean values (s.d.), medians (interquartile range) or numbers (\%).

Table 2 Comparison between patients with SBI and patients without SBI

\begin{tabular}{lccr} 
& $\begin{array}{c}\text { SBI }(+) \\
(\mathrm{n}=54)\end{array}$ & \multicolumn{1}{c}{ SBI $(-)$} & $\mathrm{P}$ \\
& $(\mathrm{n}=166)$ & \\
\hline Female sex & $15(28 \%)$ & $74(45 \%)$ & 0.038 \\
Age, years & $73(10)$ & $67(9)$ & $<0.001$ \\
CCAIMT, mm & $1.8(1.5-2.4)$ & $1.3(1.0-1.8)$ & $<0.001$ \\
ABI & $1.07(0.96-1.14)$ & $1.10(1.05-1.14)$ & 0.066 \\
CAVI & $9.5(9.2-10.9)$ & $8.8(8.0-9.7)$ & $<0.001$ \\
WMHs & $43(80 \%)$ & $59(36 \%)$ & $<0.001$ \\
Hypertension & $49(91 \%)$ & $119(72 \%)$ & 0.003 \\
Hypercholesterolemia & $27(50 \%)$ & $81(49 \%)$ & 1.000 \\
Diabetes mellitus & $14(26 \%)$ & $37(22 \%)$ & 0.582 \\
Ischemic heart disease & $24(44 \%)$ & $39(23 \%)$ & 0.005 \\
Smoking & $12(22 \%)$ & $25(15 \%)$ & 0.217 \\
& & &
\end{tabular}

Abbreviations: $\mathrm{ABI}$, ankle-brachial pressure index; CAVI, cardio-ankle vascular index; CCAIMT, common carotid artery intima-media thickness; SBI, silent brain infarct: WMHs, white-matter hyperintensities.

Data are mean values (s.d.), medians (interquartile range) or numbers (\%). 
Table 3 Comparison between patients with SBI and patients without SBI by univariable and multivariable logistic regression analyses

\begin{tabular}{|c|c|c|c|}
\hline & \multirow[b]{2}{*}{ Univariable } & \multicolumn{2}{|c|}{ Multivariable } \\
\hline & & Model 1 & Model 2 \\
\hline & OR $(95 \% \mathrm{Cl})$ & OR $(95 \% \mathrm{Cl})$ & OR $(95 \% \mathrm{Cl})$ \\
\hline Female sex & $0.56(0.29-1.06)$ & & \\
\hline Age (by one year) & $1.08(1.04-1.12)^{* *}$ & $1.08(1.03-1.13)^{*}$ & $1.07(1.03-1.12)^{*}$ \\
\hline CCAIMT, mm & $1.96(1.33-2.93)^{* *}$ & & \\
\hline CAVI $<9.2$ & & - & 1.00 (reference) \\
\hline Hypertension & $3.87(1.58-11.7)^{*}$ & & \\
\hline Hypercholesterolemia & $1.05(0.57-1.94)$ & & \\
\hline Diabetes mellitus & $1.22(0.59-2.45)$ & & \\
\hline Ischemic heart disease & $2.61(1.36-4.98)^{*}$ & $2.74(1.36-5.57)^{*}$ & $2.64(1.30-5.40)^{*}$ \\
\hline Smoking & $1.61(0.73-3.43)$ & $2.12(0.89-4.97)$ & $2.20(0.91-5.21)$ \\
\hline
\end{tabular}

Abbreviations: CAVI, cardio-ankle vascular index; CCAIMT, common carotid artery intima-media thickness; OR, odds ratio; SBI, silent brain infarct; $95 \% \mathrm{Cl}$, 95\% confidence interval.

The prevalence of SBI was the dependent variable.

$P$ shown when significant.

$P$ shown when
$*$

${ }^{* *} P<0.001$.

\section{Comparison between patients with/without WMHs}

Table 4 summarizes the clinical characteristics of patients with or without WMHs. Patients with WMHs were significantly older, had increased CAVI and CCAIMT, and were more likely to have hypertension when compared with patients without WMHs. The CAVI cutoff value for detection of WMHs was 8.9, which was associated with a $72 \%$ sensitivity and $60 \%$ specificity (AUC $=0.67 \pm 0.04$; $95 \%$ CI, 0.59 $0.74 ; P<0.001)$. In multivariable analysis (Table 5), hypertension (OR, 2.48; 95\% CI, 1.12-5.78), and age (OR, 1.17; 95\% CI, 1.12-1.23) were independently associated with WMHs.

\section{Increased CAVI and association with SBI and WMHs}

As shown in Table 6, lower CAVI indicated the absence of both SBI and WMHs. However, this association lost statistical significant after adjustment for age and sex. Patients with CAVI $\geqslant 9.2$ had a higher probability of the presence of SBI (OR, 2.55), and both SBI and WMHs (OR, 2.57) when compared with patients with CAVI $<9.2$ after adjustment for age and sex.

\section{DISCUSSION}

The main finding of the present study was that CAVI is independently associated with SBI. However, CAVI was not independently associated with WMHs. Furthermore, the present study suggests that there is a strong association between SBI and arterial stiffness.

The independent association between SBI and CAVI in the present study is in agreement with previous reports regarding SBI and arterial stiffness indicated by aortic PWV and baPWV. ${ }^{2,13,14,27}$ Indeed, SBI is considered to be related to cerebral microvascular arteriolosclerosis such as lipohyalinosis and microatheroma, which provides a rational basis for the independent associations between SBI and both baPWV and CAVI as parameters of arterial stiffness. ${ }^{5-7,9-13}$ Furthermore, SBI was independently associated with IHD in this cohort. In general, atherosclerosis involves a combination of fatty degeneration (atherosis) and vessel stiffing (sclerosis). ${ }^{19}$ Similarly, the etiology of IHD is mainly coronary atherosclerosis or arteriosclerosis, including atheromatous changes and calcification, and the independent association between coronary artery calcification and cerebral SVD has been reported previously. ${ }^{8,21}$ Hence, this association supports the relationship between IHD and SBI in the present study.
Table 4 Comparison between patients with WMHs and patients without WMHs

\begin{tabular}{lccr}
\hline & $\begin{array}{c}\text { WMHs }(+) \\
(\mathrm{n}=102)\end{array}$ & $\begin{array}{c}\text { WMHs }(-) \\
(\mathrm{n}=118)\end{array}$ & $\mathrm{P}$ \\
\hline Female sex & $43(42 \%)$ & $46(39 \%)$ & 0.680 \\
Age, years & $74(7)$ & $64(9)$ & $<0.001$ \\
CCAIMT, mm & $1.7(1.3-2.2)$ & $1.3(1.0-1.8)$ & $<0.001$ \\
ABI & $1.08(1.04-1.13)$ & $1.10(1.05-1.14)$ & 0.190 \\
CAVI & $9.4(8.6-10.4)$ & $8.7(7.8-9.4)$ & $<0.001$ \\
SBI & $43(80 \%)$ & $11(9 \%)$ & $<0.001$ \\
Hypertension & $89(87 \%)$ & $79(67 \%)$ & $<0.001$ \\
Hypercholesterolemia & $49(48 \%)$ & $59(50 \%)$ & 0.788 \\
Diabetes mellitus & $37(26 \%)$ & $24(20 \%)$ & 0.337 \\
Ischemic heart disease & $30(29 \%)$ & $33(28 \%)$ & 0.881 \\
Smoking & $15(15 \%)$ & $22(19 \%)$ & 0.474 \\
\hline Abbrevitions: & & & \\
\hline
\end{tabular}

Abbreviations: $\mathrm{ABI}$, ankle-brachial pressure index; CAVI, cardio-ankle vascular index; CCAIMT, common carotid artery intima-media thickness; SBI, silent brain infarct; WMHs, white-matter hyperintensities.

Data are mean values (s.d.), medians (interquartile range) or numbers (\%).

Table 5 Comparison between patients with WMHs and patients without WMHs by univariable and multivariable logistic regression analyses

\begin{tabular}{lll}
\hline & \multicolumn{1}{c}{$\begin{array}{c}\text { Univariable } \\
\text { OR }(95 \% \mathrm{Cl})\end{array}$} & $\begin{array}{c}\text { Multivariable } \\
\text { OR }(95 \% \mathrm{Cl})\end{array}$ \\
\hline Female sex & $1.29(0.75-2.21)$ & \\
Age (by one year) & $1.17(1.12-1.23)^{* *}$ & $1.17(1.12-1.23)^{* *}$ \\
CCAIMT, mm & $1.68(1.17-2.48)^{*}$ & \\
CAVI (every one point increase) & $1.42(1.18-1.74)^{* *}$ & \\
Hypertension & $3.38(1.72-7.00)^{* *}$ & $2.48(1.12-5.78)^{*}$ \\
Hypercholesterolemia & $0.92(0.54-1.57)$ & \\
Diabetes mellitus & $1.41(0.75-2.66)$ & \\
Ischemic heart disease & $1.07(0.60-1.93)$ & \\
Smoking & $0.75(0.36-1.53)$ & \\
\hline
\end{tabular}

Abbreviations: CAVI, cardio-ankle vascular index; CCAIMT, common carotid artery intima-media thickness; OR, odds ratio; SBI, silent brain infarct; WMHs, white-matter hyperintensities; $95 \% \mathrm{Cl}, 95 \%$ confidence interval.

The prevalence of WMHs was the dependent variable.

$P$ shown when significant.

${ }^{*} P<0.05$.

${ }^{* *} P<0.001$. 
Table 6 Comparison of ORs for the presence of SBI and WMHs

\begin{tabular}{|c|c|c|c|}
\hline ORs $(95 \% \mathrm{Cl})$ & SBI (-) and WMHs (-) & $S B I(+)$ & SBI (+) and WMHs (+) \\
\hline CAVI (every one point increase) ${ }^{a}$ & $0.73(0.59-0.87)^{* * *}$ & $1.43(1.19-1.76)^{* * *}$ & $1.58(1.28-1.99)^{* * *}$ \\
\hline CAVI (every one point increase) ${ }^{b}$ & $0.94(0.77-1.15)$ & $1.26(1.03-1.57)^{* *}$ & $1.33(1.06-1.70)^{*}$ \\
\hline $\mathrm{CAVI} \geqslant 9.2^{\mathrm{a}}$ & $0.34(0.19-0.59)^{* * *}$ & $3.85(2.02-7.56)^{* * *}$ & $5.01(2.43-11.03)^{* * *}$ \\
\hline $\mathrm{CAVI} \geqslant 9.2^{\mathrm{b}}$ & $0.74(0.39-1.43)$ & $2.55(1.26-5.29)^{* *}$ & $2.57(1.15-5.98)^{*}$ \\
\hline
\end{tabular}

Abbreviations: CAVI, cardio-ankle vascular index; OR, odds ratio; SBI, silent brain infarct; WMHs, white-matter hyperintensities; 95\% $\mathrm{Cl}$, 95\% confidence interval.

aUnadjusted OR.

${ }^{\mathrm{b}}$ Adjusted OR for age and sex.

${ }^{*} P<0.05$.

$* * * P<0.001$

The association between WMHs and CAVI remains controversial. Previous reports have demonstrated that WMHs are independently associated with baPWV, aortic PWV and central systolic blood pressure. ${ }^{7,13,17,27}$ Although we confirmed that there was a significantly increased CAVI in patients with WMHs when compared with those without WMHs, as has been reported previously, ${ }^{23}$ CAVI was not independently associated with WMHs in the present study. This might arise from the fact that the majority of our cohort comprising those patients with hypertension and WMHs or because the present study treated periventricular hyperintensity and deep white-matter hyperintensity as WMHs. Previous reports have demonstrated that baPWV is an independent factor for the presence of periventricular hyperintensity, but not deep white-matter hyperintensity. ${ }^{18}$ Alternatively, SBI and WMHs may represent different forms of SVD. ${ }^{2,10-12}$ Indeed, SBI is related to arteriolosclerosis such as lipohyalinosis and microatheroma, while WMHs are associated with the extravasation of toxic plasma constituents into the cerebral parenchyma due to endothelial dysfunction..$^{8-12}$ Finally, CAVI is independent of blood pressure because of the adjustment of blood pressure based on a stiffness parameter $\beta$, which is an index of local stiffness of a blood vessel, ${ }^{20-23}$ and the stiffness parameter $\beta$ may not be associated with WMHs. In the present study, both hypertension and age were independently associated with WMHs, which is consistent with observations from previous reports. ${ }^{17}$ This suggests that hypertension and age are more important in the pathogenesis of WMHs when compared with SBI. Hatanaka et al. ${ }^{13}$ also reported that the association between arterial stiffness and WMHs was not robust when compared with the association between arterial stiffness and SBI.

The CAVI cutoff values for SBI and WMHs were roughly equal (9.2 and 8.9, respectively). Previous reports suggest that the CAVI cutoff value for IHD is 9.20,22 Although CAVI was not independently associated with WMHs in the present study, this concurrence of CAVI cutoff values suggests that both SBI and WMHs are related to an arteriosclerotic etiology. However, on the basis of our results, a stiffness parameter $\beta$ may be a differential factor between SBI and WMHs in the view of arterial stiffness.

The possibility of the presence of both SBI and WMHs is at least 2.5 -fold higher in patients with CAVI $\geqslant 9.2$ than in patients with CAVI $<9.2$, as shown in Table 6 . Thus, we recommend the use of brain MRI to detect SVD in patients with CAVI $\geqslant 9.2$ (otherwise $\geqslant 9$ ), even if they are neurologically asymptomatic. Likewise, patients with IHD could be recommended for brain imaging because of the independent association between SBI and IHD. Since stroke is a major public health problem, assessment of the risk of stroke is especially important. ${ }^{4,7}$ Assessing CAVI may be reasonable, convenient and cost effective as an initial assessment tool to identify SVD, and also baPWV.

CCAIMT, the other parameter of arterial stiffness, ${ }^{17,34}$ significantly correlated with increased CAVI in our study (data not shown). This correlation indicates that both CCAIMT and CAVI are associated with early atherosclerotic changes (arterial stiffness). ${ }^{21,34}$ Carotid intima-media thickness is also a useful and non-invasive method to examine atherosclerosis and is associated with future stroke risk. ${ }^{2,3,34}$

There were several limitations to this study. Selection bias is possible, because this was a hospital-based cohort study, and we mainly enrolled patients who were relatively old and had cardiovascular risk factors. Similarly, causal relationships between the factors and both SBI and WMHs remain unclear, because this was a correlational study. T1-weighted imaging in addition to T2WI and FLAIR images is desirable for more accurate detection of SBI. However, the averaged AUC for SBI in the combination of T2WI and FLAIR images (0.85) was similar to that of all the three image types (0.95). ${ }^{35}$ Finally, other factors may also be associated with SBI and $\mathrm{WMHs}$, including central systolic blood pressure, chronic kidney disease and cerebral microbleeds. ${ }^{2,10,11,15,17,25,30}$ Further research is needed to validate an appropriate stroke prevention strategy for patients with both SBI and WMHs.

In conclusion, measurement of CAVI can indicate the probability of the presence of SBI and may identify patients at potential risk for stroke.

1 Vlachopoulos C, Aznaouridis K, Stefanadis C. Prediction of cardiovascular events and all-cause mortality with arterial stiffness. J Am Coll Cardiol 2010; 55: 1318-1327.

$2 \mathrm{Kim} \mathrm{DH}, \mathrm{Kim} \mathrm{J}, \mathrm{Kim} \mathrm{JM}$, Lee AY. Increased brachial-ankle pulse wave velocity is independently associated with risk of cerebral ischemic small vessel disease in elderly hypertensive patients. Clin Neurol Neurosurg 2008; 110: 599-604.

3 Matsumoto M, Inoue K, Moriki A. Associations of brachial-ankle pulse wave velocity and carotid atherosclerotic lesions with silent cerebral lesions. Hypertens Res 2007; 30: 767-773.

4 Saji N, Shimizu H, Kawarai T, Tadano M, Kita Y, Yokono K. Clinical features of a firstever lacunar infarction in Japanese patients: poor outcome in females. J Stroke Cerebrovasc Dis 2011; 20: 231-235.

5 Bokura H, Kobayashi S, Yamaguchi S, lijima K, Nagai A, Toyoda G, Oguro H, Takahashi K. Silent brain infarction and subcortical white matter lesions increase the risk of stroke and mortality: a prospective cohort study. J Stroke Cerebrovasc Dis 2006; 15: 57-63.

6 Vermeer SE. Silent brain infarcts and white matter lesions increase stroke risk in the general population: the Rotterdam scan study. Stroke 2003; 34: 1126-1129.

7 Saji N, Shimizu H, Kawarai T, Tadano M, Kita Y, Yokono K. Increased brachial-ankle pulse wave velocity is independently associated with white matter hyperintensities. Neuroepidemiology 2011; 36: 252-257.

8 Kim BJ, Lee SH, Kim CK, Ryu WS, Kwon HM, Choi SY, Yoon BW. Advanced coronary artery calcification and cerebral small vessel diseases in the healthy elderly. Circ $J$ 2011; 75: 451-456.

9 Wardlaw JM. Blood-brain barrier and cerebral small vessel disease. J Neurol Sci 2010; 299: 66-71.

10 Pantoni L. Cerebral small vessel disease: from pathogenesis and clinical characteristics to therapeutic challenges. Lancet Neurol 2010; 9: 689-701.

11 Patel B, Markus HS. Magnetic resonance imaging in cerebral small vessel disease and its use as a surrogate disease marker. Int J Stroke 2011; 6: 47-59.

12 Lim JS, Kwon HM. Risk of 'silent stroke' in patients older than 60 years: risk assessment and clinical perspectives. Clin Interv Aging 2010; 5: 239-251. 
13 Hatanaka R, Obara T, Watabe D, Ishikawa T, Kondo T, Ishikura K, Aikawa T, Aono Y, Hara A, Metoki H, Asayama K, Kikuya M, Mano N, Ohkubo T, Izumi S, Imai Y. Association of arterial stiffness with silent cerebrovascular lesions: the Ohasama study. Cerebrovasc Dis 2011; 31: 329-337.

14 Saji N, Kimura K, Shimizu H, Kita Y. Association between silent brain infarct and arterial stiffness indicated by brachial-ankle pulse wave velocity. Intern Med 2012 (in press).

15 Otani H, Kikuya M, Hara A, Terata S, Ohkubo T, Kondo T, Hirose T, Obara T, Metoki H, Inoue R, Asayama K, Kanno A, Terawaki H, Nakayama M, Totsune K, Hoshi H, Satoh H, Izumi S-I, Imai Y. Association of kidney dysfunction with silent lacunar infarcts and white matter hyperintensity in the general population: the Ohasama study. Cerebrovasc Dis 2010; 30: 43-50.

16 Yao H, Miwa Y, Takashima Y, Yahara K, Hashimoto M, Uchino A, Yuzuriha T, Sasaguri T. Chronic kidney disease and subclinical lacunar infarction are independently associated with frontal lobe dysfunction in community-dwelling elderly subjects: the Sefuri brain MRI study. Hypertens Res 2011; 34: 1023-1028.

17 Shrestha I, Takahashi T, Nomura E, Ohtsuki T, Ohshita T, Ueno H, Kohriyama T, Matsumoto M. Association between central systolic blood pressure, white matter lesions in cerebral MRI and carotid atherosclerosis. Hypertens Res 2009; 32: 869-874.

18 Ohmine T, Miwa Y, Yao H, Yuzuriha T, Takashima Y, Uchino A, Takahashi-Yanaga F, Morimoto S, Maehara Y, Sasaguri T. Association between arterial stiffness and cerebral white matter lesions in community-dwelling elderly subjects. Hypertens Res 2008; 31 : 75-81.

19 Okura T, Watanabe S, Kurata M, Manabe S, Koresawa M, Irita J, Enomoto D, Miyoshi K, Fukuoka T, Higaki J. Relationship between cardio-ankle vascular index (CAVI) and carotid atherosclerosis in patients with essential hypertension. Hypertens Res 2007; 30: 335-340.

20 Horinaka S, Yabe A, Yagi H, Ishimura K, Hara H, lemua T, Matsuoka H. Comparison of atherosclerotic indicators between cardio ankle vascular index and brachial ankle pulse wave velocity. Angiology 2009; 60: 468-476.

21 Izuhara M, Shioji K, Kadota S, Baba O, Takeuchi Y, Uegaito T, Mutsuo S, Matsuda M. Relationship of cardio-ankle vascular index (CAVI) to carotid and coronary arteriosclerosis. Circ J 2008; 72: 1762-1767.

22 Shirai K. Cardia-ankle vascular index (CAVI) as a novel indicator of arterial stiffness: theory, evidence and perspectives. J Atheroscler Thromb 2011; 18: 924-938.

23 Suzuki J, Sakakibara R, Tomaru T, Tateno F, Kishi M, Ogawa E, Kurosu T, Shirai K. Stroke and cardio-ankle vascular stiffness index. J Stroke Cerebrovasc Dis 2011 (in press).

24 Yamamoto N, Yamanaka G, Ishikawa M, Takasugi E, Murakami S, Yamanaka T, Ishine M, Matsubayashi K, Hanafusa T, Otsuka K. Cardio-ankle vascular index as a predictor of cognitive impairment in community-dwelling elderly people: four-year follow-up. Dement Geriatr Cogn Disord 2009; 28: 153-158.
25 Laurent S, Cockcroft J, Van Bortel L, Boutouyrie P, Giannattasio C, Hayoz D, Pannier B, Vlachopoulos C, Wilkinson I, Struijker-Boudier H. Expert consensus document on arterial stiffness: methodological issues and clinical applications. Eur Heart J 2006; 27: 2588-2605.

26 Turin TC, Kita Y, Rumana N, Takashima N, Kadota A, Matsui K, Sugihara H, Morita Y, Nakamura Y, Miura K, Ueshima H. Brachial-ankle pulse wave velocity predicts all-cause mortality in the general population: findings from the Takashima study, Japan. Hypertens Res 2010; 33: 922-925.

27 Henskens LH, Kroon AA, Van Oostenbrugge RJ, Gronenschild EH, Fuss-Lejeune MM, Hofman PA, Lodder J, de Leeuw PW. Increased aortic pulse wave velocity is associated with silent cerebral small-vessel disease in hypertensive patients. Hypertension 2008; 52: 1120-1126.

28 Terai M, Ohishi M, Ito N, Takagi T, Tatara Y, Kaibe M, Komai N, Rakugi H, Ogihara T. Comparison of arterial functional evaluations as a predictor of cardiovascular events in hypertensive patients: the Non-Invasive Atherosclerotic Evaluation in Hypertension (NOAH) study. Hypertens Res 2008; 31: 1135-1145.

29 Ohishi M, Tatara Y, Ito N, Takeya Y, Onishi M, Maekawa Y, Kato N, Kamide K, Rakugi H. The combination of chronic kidney disease and increased arterial stiffness is a predictor for stroke and cardiovascular disease in hypertensive patients. Hypertens Res 2011; 34: 1209-1215.

30 Ochi N, Kohara K, Tabara Y, Nagai T, Kido T, Uetani E, Ochi M, Igase M, Miki T. Association of central systolic blood pressure with intracerebral small vessel disease in Japanese. Am J Hypertens 2010; 23: 889-894.

31 Schmidt R, Fazekas F, Kapeller P, Schmidt H, Hartung HP. MRI white matter hyperintensities: three-year follow-up of the Austrian Stroke Prevention Study. Neurology 1999; 53: 132-139.

32 Fazekas F, Kleinert R, Offenbacher H, Schmidt R, Kleinert G, Payer F, Radner H, Lechner H. Pathologic correlates of incidental MRI white matter signal hyperintensities. Neurology 1993; 43: 1683-1689.

33 Katakami N, Kim YS, Kawamori R, Yamasaki Y. The phosphodiesterase inhibitor cilostazol induces regression of carotid atherosclerosis in subjects with type 2 diabetes mellitus: principal results of the Diabetic Atherosclerosis Prevention by Cilostazol (DAPC) study: a randomized trial. Circulation 2010; 121: 2584-2591.

34 Tzortzis S, Ikonomidis I, Lekakis J, Papadopoulos C, Triantafyllidi H, Parissis J, Trivilou P, Paraskevaidis I, Anastasiou-Nana M, Kremastinos DT. Incremental predictive value of carotid intima-media thickness to arterial stiffness for impaired coronary flow reserve in untreated hypertensives. Hypertens Res 2010; 33: 367-373.

35 Sasaki M, Hirai T, Taoka T, Higano S, Wakabayashi C, Matsusue E, Ida M. Discriminating between silent cerebral infarction and deep white matter hyperintensity using combinations of three types of magnetic resonance images: a multicenter observer performance study. Neuroradiology 2008; 50: 753-758. 